
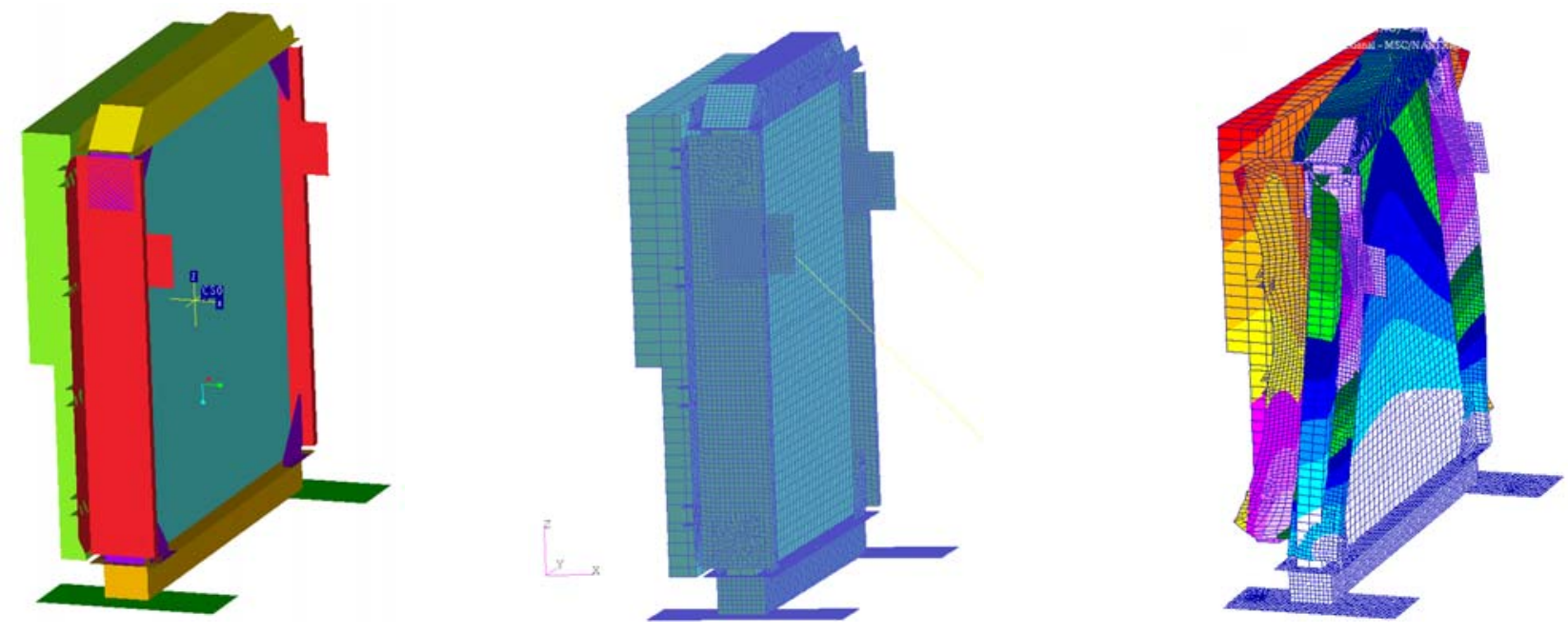

\title{
Product Data Quality and Collaborative Engineering
}

\author{
Manuel Contero ${ }^{*}$, Pedro Company ${ }^{\dagger}$, \\ Carlos Vila ${ }^{\dagger}$, Nuria Aleixos ${ }^{\dagger}$

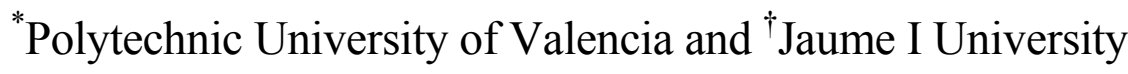

Front-page photo: The left image represents an automotive radiator model simplified for FEM analysis. The central image shows the mesh model, and the right image represents modal analysis results

\section{Abstract}

In this paper, the impact of product data quality in the implementation of collaborative engineering in an extended enterprise framework is analyzed. Previously, some definitions about collaborative and concurrent engineering and present concepts like extended and/or virtual enterprise, digital mock-up, virtual prototype and virtual factory are reviewed.

The product data model as a key element for the product development process is analyzed. The different views of this model are placed according to the fields where they apply. The importance of product model quality in the current status of data exchange standards is highlighted, with particular attention to ISO 10303 (STEP) new developments. Current state-of-art on data quality models, and product data quality recommendations such as VDA 4955/2 and SASIG PDQ, are also revised. Finally, our own product data quality model is presented. This comprises three points of view or levels: morphological, syntactic and semantic. Hence, it provides a tool for a better understanding of product data quality that helps find solutions that avoid the interoperability problem. Throughout the paper, references to the automotive industry will be used to illustrate concepts. 


\section{Introduction}

Product Development is a key activity for enterprise survival and competitiveness. This process must be agile and efficient so as to provide enough flexibility to adapt to a changing market. Most new Product Development methods are based on empowering the role of design and shortening the development cycle of new products. Digital tools like CAX and Product Data Management (PDM) systems are key elements in this strategy. They allow product developers to experiment with many alternative solutions, providing better products with better quality, in less time and at a lower cost. Shortening the development cycle and lowering cost are some of the advantages of employing digital mock-ups and simulating the manufacturing process in a virtual environment.

A complete digital representation of the product and its manufacturing process allows complex simulations to be carried out, avoiding the construction of physical prototypes and providing early detection of bottlenecks in the manufacturing process. In this way, an important time reduction in the whole development process as well as better quality are obtained, because more design alternatives can be explored. However, this approach is not exempt of problems, because it is necessary to transfer product data between different software applications. This introduces the data exchange problem, because data can be lost or degenerated during exchanges. In this context, product data quality is becoming a key issue to guarantee a true integration among participants defining the product development process.

\section{Concurrent Engineering}

Product Development has suffered an enormous evolution during the last two decades. The appearance of Concurrent Engineering (CE) was a milestone in simultaneously lowering product cost, increasing product quality and reducing time to market. Concurrent Engineering began as an initiative of the US Department of Defense. In 1982, the Defense Advanced Research Projects Agency (DARPA) began a program with the objective of improving Product Development. As a result of this program, Winner et al. [1] first defined the term Concurrent Engineering as "... a systematic approach to the integrated, concurrent design of products and their related processes, including manufacturing and support. This approach is intended to cause the developers, from the outset, to consider all elements of the product life-cycle from conception to disposal, including quality cost, schedule, and user requirements."

After this project, the DARPA started a five-year program: the DARPA Initiative in Concurrent Engineering (DICE), aimed to incorporate this methodology in the US military industry. As part of this initiative, the "Concurrent Engineering Research Center" (CERC) was founded at West Virginia University in the US. As a result of this work, Cleetus [2] proposed another definition for CE: "Concurrent Engineering is a systematic approach to the integrated and concurrent development of a product and its related processes, that emphasizes response to customer expectations and embodies team values of cooperation, trust, and sharing in such a manner that decision making proceeds with large intervals of parallel working by all life-cycle perspectives, synchronized by comparatively brief exchanges to produce consensus."

\section{Extended and Virtual Enterprise}

At the end of 90's the quest for reducing costs lead to the progressive outsourcing of design tasks to suppliers. This movement brought suppliers into greater involvement in design and product technology responsibility [3]. The most advanced industries, like the automotive, aeronautical and aerospace ones, soon adopted this trend. Automotive maker Chrysler pioneered the development and use of the Extended Enterprise concept. It means working closely with the supply base in a teamwork atmosphere of cooperation based in trust, communication and partnership, where the workgroup is usually geographically dispersed and advanced tools support communications.

In recent years, new enterprise models appear to exploit modern high-performance computer networks. In this context, the concept of Virtual Enterprise [4] with its sharing of data, costs, skills, and technology allows this new kind of enterprise to put products in the market that they could not previously deliver individually. The European Society of Concurrent Engineering [5] defines a Virtual Enterprise as a "distributed, temporary alliance of independent, co-operating companies in the design and manufacturing of products and services. Such a complex organization makes use of systematic approaches, methods and advanced technologies for increasing efficiency, and is enacted by the means offered by recent Information and Communication Technologies".

\section{Concurrent Enterprise}

Integrating the Virtual Enterprise paradigm and the methods of Concurrent Engineering, a new concept named Concurrent Enterprise arises. Thoben and Weber [6] proposed the following definition: "The Concurrent Enterprise is a distributed, temporary alliance of independent, co-operating manufacturers, customers and suppliers using systematic approaches, methods and advanced technologies for increasing efficiency in the design and manufacturing of products (and services) by means of parallelism, integration, team work, etc. for achieving common goals on global markets."

\section{Collaborative Engineering}

The scope of Concurrent Engineering must be broaden to include the new models of "Extended Enterprise", "Virtual enterprise" and "Concurrent enterprise" that have become commonplace during the last decade. The concept of Collaborative Engineering encompasses both supplier integration and advanced communications tools to cope with the product development process and extends the scope of Concurrent Engineering. With the intention of widening the 
scope of Concurrent Engineering, de Graaf [7] proposes the following definition for Collaborative Engineering: "Collaborative Engineering is a systematic approach to control life-cycle cost, product quality and time to market during Product Development, by concurrently developing products and their related processes with response to customer expectations, where decision making ensures input and evaluation by all life-cycle disciplines, including suppliers, and information technology is applied to support information exchange where necessary."

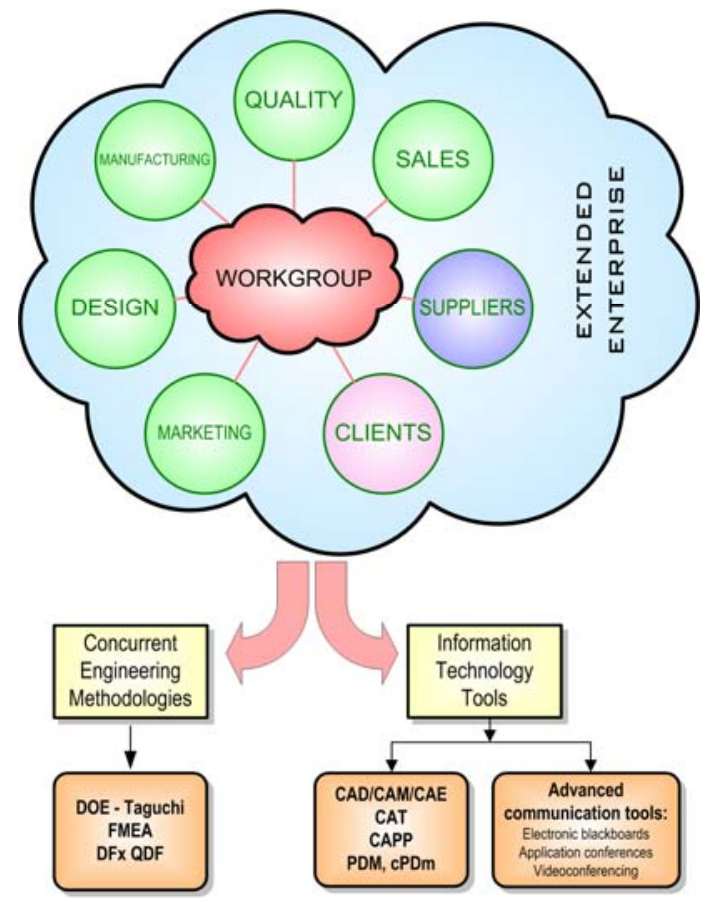

Figure 1. Collaborative Engineering model.

In Figure 1, a schematic vision of our Collaborative Engineering model is presented, based on de Graaf's definition. The central element is the workgroup, usually geographically dispersed, working in the context of the Extended and/or Virtual Enterprise. Concurrent Engineering methodologies and Information Technologies tools support the Product and Processes Development. As in de Graaf's definition, product life cycle, customer input and supplier involvement are underlying elements included in the model.

\section{Concurrent Engineering Methodologies}

As noted in the Collaborative Engineering definition, the virtual workgroup employs Concurrent Engineering methodologies $[8,9]$. Some of the more frequently used ones are:

- QFD (Quality Function Deployment) a structured method in which customer requirements are translated into appropriate technical requirements for each stage of product development and production.
- DfX (Design for $\mathrm{X}$ ) techniques capture, in a standard procedure, all the factors known to be important in a particular design activity. For example:

$\circ$ Design for Manufacturability (DfM): rules that can ease manufacturing during early conceptual development.

- Design for Assembly (DfA): rules that can ease assembly during early conceptual development.

- Design for Environment (DfE): rules to achieve a design that uses minimum material and energy at all stages of its life cycle providing maximum reuse and recycling of products.

- FMEA (Failure Model and Effects Analysis): a procedure by which each potential failure mode in a system is analyzed to determine the potential effects caused on the system and to classify each potential failure mode according to its severity.

- DOE (Design of Experiments): a branch of applied statistics dealing with planning, conducting, analyzing, and interpreting controlled tests to evaluate the factors that control the value of a parameter or group of parameters.

- Taguchi methods: a quality engineering methodology, based on the design of experiments to provide near optimal quality characteristics for a specific objective to improve quality and reduce costs.

\section{Information Technology Tools}

Information Technology (IT) development has completely transformed the Product Development. New methodologies, specifically oriented toward shortening the development cycle, have been adopted. The present growth in simulationbased design tools makes it possible analyzing the behavior of complex products without constructing physical prototypes. Virtual factory software permits production to be simulated, and bottlenecks to be detected early in the factory design phase. These new methods are represented in Figure 2. The essential element in this development approach is the 3D solid model provided by CAD applications. A plethora of downstream applications like CAM, CAE and many other CAX tools depends on the geometric model.

Digital Mock-Up (DMU) tools are able to manage large assemblies of thousands of parts. In this way, it is possible to detect tolerance and assembly problems early in the design phase. Current DMU applications are capable of managing complex products such as a complete airplane representation. However, optimized tessellated representations extracted from the $3 \mathrm{D}$ solid models are needed to cope with so many parts. Some systems also provide several representations for each part, each one according to a different Level of Detail (LOD). These tools provide simultaneous capabilities for design collaboration, mark up, fly through, and interference and collision detection.

Virtual Prototyping tools go a step beyond. Their objective is to assess product function and operating performance. Virtual Prototyping solutions make use of finite element analysis and advanced calculus to accurately predict 
the operating performance of the product by means of virtual tests. Thus, we can simulate a crash test with a virtual car, analyze its dynamic behavior, optimize aerodynamics with computational fluid dynamics (CFD) applications, and so on.

In the superior stage, Virtual Factory Simulation [10] is used to assess manufacturability and assembly of the product. There are two main types of simulations:

- Discrete event simulation (DES) applications simulate the behavior of entities when an event occurs at a distinct time. This kind of simulation is aimed at material flow simulation, manufacturing system and information flow analysis. Usually, time in a DES simulator does not proceed linearly but in irregular intervals.

- Geometric simulation GS, also known as continuous simulation, proceeds with time linearly in constant intervals, and provides a geometric representation of the whole manufacturing system. It is appropriate for 3-D visualization, off-line programming of robots and collision detection during manufacturing process.

\begin{tabular}{|c|c|c|c|}
\hline \multicolumn{4}{|c|}{ VIRTUAL FACTORY } \\
\hline \multicolumn{3}{|c|}{ VIRTUAL PROTOTYPE } & \multirow{4}{*}{$\begin{array}{l}\text { Production } \\
\text { Logistic } \\
\text { Costs } \\
\text { Marketing }\end{array}$} \\
\hline \multicolumn{2}{|c|}{ DMU } & \multirow{4}{*}{$\begin{array}{l}\text { Functional } \\
\text { analysis } \\
\text { Global } \\
\text { behaviour } \\
\text { analysis }\end{array}$} & \\
\hline CAD & \multirow{3}{*}{$\begin{array}{l}\text { Large } \\
\text { assembly } \\
\text { analysis } \\
\text { Fitting } \\
\text { analysis } \\
\text { Product } \\
\text { structure }\end{array}$} & & \\
\hline $\begin{array}{c}\text { Geometry } \\
\text { Features } \\
\text { Parametric data } \\
\text { Part analysis } \\
\text { Assembly analysis }\end{array}$ & & & \\
\hline & & & \\
\hline
\end{tabular}

\section{Figure 2. Advanced product development.}

Virtual Factory Simulation provides significant savings, allowing early detection of manufacturing bottlenecks in the design phase, not under operation.

\section{PMD and $\mathrm{cPDm}$}

Product Data Management (PDM) [11] is the supporting tool that enables these advanced simulations to be performed. PDM has evolved from a mid-80's CAD file manager application to provide sophisticated functions:

- $\quad$ Engineering Data Management: providing data vaulting and document management, product structure and configuration management, classification and search.

- Engineering Workflow Management: providing project management, engineering change and release management and communication support.

At present, PDM systems are evolving to take into account Internet, Web-based technologies, and the new extended and/or virtual enterprise paradigm. This evolution leads to the concept of "collaborative Product Definition management" (cPDm) [12], which is a broadening of PDM capabilities to support the management of product definition and associated processes in the extended enterprise framework by means of Internet/Web technologies. Systems such as these are particularly interesting for global companies with facilities located around the world and also for enabling true integration among OEMs, clients and suppliers in the product development process.

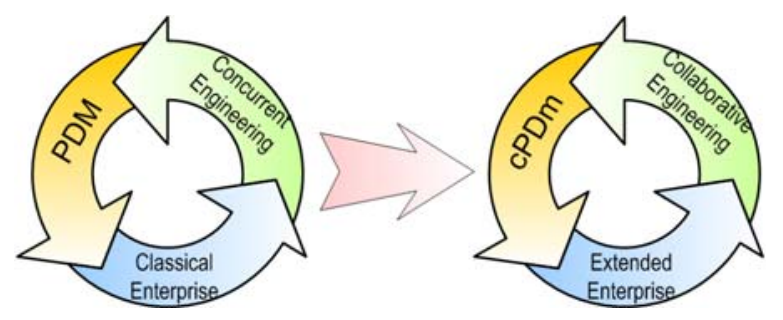

Figure 3. Evolution from PDM to cPDm

\section{Web-based CAD and Communication Tools}

The heterogeneous enterprise architectures presented previously encouraged the development of new Web-based design tools, which combine CAD, PDM and Web access in a unified environment. These tools are aimed at reducing costs between Original Equipment Manufactures (OEMs) and suppliers sharing a common design platform. Usually these kind of applications are built on a 3-tier architecture using Internet as the communication infrastructure. Thus, we have a first tier where a Thin Client, usually through an Internet navigator, provides the front-end to the system. In a second tier, an Application Server hosts the software application. Finally, the Database Server holding the central data repository that stores and manages design data provides the third tier. This technology also introduces the concept of subscription, where users pay a monthly subscription fee for the service. This approach allows companies to reduce information technology expenses by avoiding the need to buy and maintain expensive software and hardware. The growing Internet bandwidth is supposed to broaden this technology in the near future.

Finally, Communication Tools as supporting technologies for Collaborative Engineering will be discussed. These tools are evolving parallel to Internet, and are fundamental to providing collaboration for a geographically dispersed work team. A distinction can be done between synchronous and asynchronous collaboration [13], depending on whether the collaborative partners are working simultaneously or not. Examples of asynchronous collaboration are e-mail and newsgroups. On the other hand, to arrange a virtual meeting between the partners, synchronous communication tools are needed; such as whiteboards, videoconferencing and application sharing. In the context of the extended enterprise, it is usual to find a multi-platform and multi-vendor environment. For that reason, communication standards are an enabling element to real team collaboration. The International Telecommunication Union and the International Multimedia Teleconferencing Consortium have developed several 
families of standards for this purpose. Thus, the T.120 Series of Recommendations collectively define a multipoint data communication service for use in multimedia conferencing environments. Inside this series, Recommendations related to the communication layer are found (T.122, T.123 and T.125). The collaboration layer provides support for both data and audio/video conference. The recommendations related to data conferencing are:

- T.126: Multipoint still image and annotation protocol.

- T.127: Multipoint binary file transfer protocol.

- T.128: Multipoint application sharing.

- T.134: Text chat application entity.

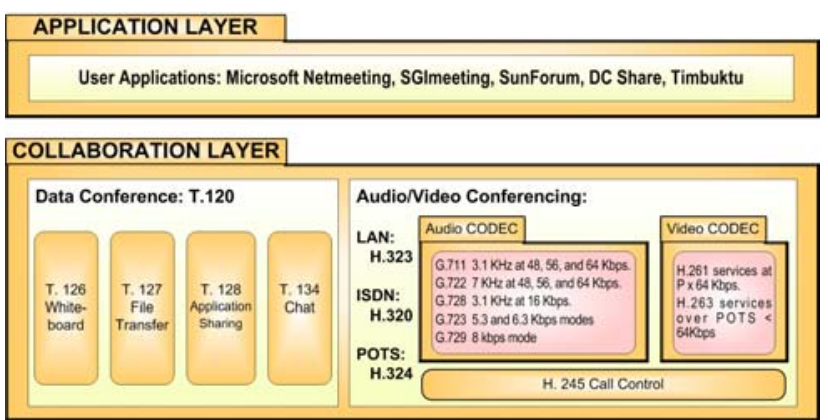

COMMUNICATION LAYER

Multi-point communication service and network-specific transport protocol: T.122 T.123 T.125 Internet / Intranet over: LAN, ISDN or POTS

Figure 4. Architecture of communication tools.

The Audio/Video conferencing part proposes three standards associated with communication bandwidth:

- H.320 for ISDN videoconferencing.

- H.323 for LAN videoconferencing.

- H.324 for low bit rate connections such as POTS.

Nowadays, the main limitation for the use of these tools is communication bandwidth. From a practical point of view, in restricted bandwidth situations parts of the data-video-audio conference can be redirected to other communication channels; for instance, moving audio conferencing to normal telephone calls, and making a selective use of the video, which is the most bandwidth consuming part.

One of the most interesting facts about communications tools is that many of them are free, or have a very reduced cost. Thus, an imaginative use of them can be very productive. For example, setting up a newsgroup server can be a very inexpensive way to provide a discussion forum where work team members can ask for help or receive general notifications about the product development process.

To finish this analysis of Collaborative Engineering, it must be emphasized that the key for all the Product Development Process is a digital product representation. The next section will study this aspect in depth.

\section{Product Data Model}

CAD and PDM systems are the primary elements for the Advanced Product Development Process, as noted in Figure 2. Product Data Management Systems [11,14] supply an infrastructure oriented to provide everybody's need of information in a concurrent engineering environment. These systems also cover external partners' access, and company security and release procedures. The following can be distinguished:

- Product data (and tooling data): geometry, DMU, analysis and simulation results, materials, reports, etc.

- Process data: advanced manufacturing engineering data (relations between parts/tools/processes), build sequence planning and machining data, work cell definition and plant layout, and so on

Both types of data are closely related to the geometric model provided by CAD applications. As will be seen later, the quality of these CAD models will be of vital importance for a smooth integration among the participants of the Product Development Process.

\section{Primary and Secondary Views}

From a practical point of view, as this analysis is restricted to the available commercial technology, we propose the Product Data Model represented in Figure 5. This model is built on a PDM system, which serves as the repository of the different product views that integrate the Digital Product Master Model. CAD provides the connection line among those different views.

Current technology is clearly biased towards Design, [16]. Hence, the 3D solid models are considered as the Primary View, deriving Secondary Views for other purposes like DMU, analysis or manufacturing. Any modification of the geometry must be performed on the Primary View. The way in which the different tasks in the Advanced Product Development Process make use of the Primary View will now be analyzed:

- Documentation: Most of the Engineering Drawings are obtained from the 3D geometric model. Projections and sections are easily created from the 3D model. Many parametric systems propose a set of dimensions, and the user has only to select the more convenient ones. Nevertheless, in the near future, we expect the drawings to be relegated to a secondary role. They will be even eliminated, at least in the most technological advanced industries; in step 3 of VDA 4953 Recommendation [15] the creation of drawings is omitted. The legal implications should be noted, since OEMs assign a binding nature to $\mathrm{CAD}$ model data [17].

- Rendering: this application takes advantage of the 3D model by means of a surface representation generated by a tessellation process, as provided by the stereolithography STL format.

- Digital mock-ups: This application usually uses simplified representations of parts, obtained by 


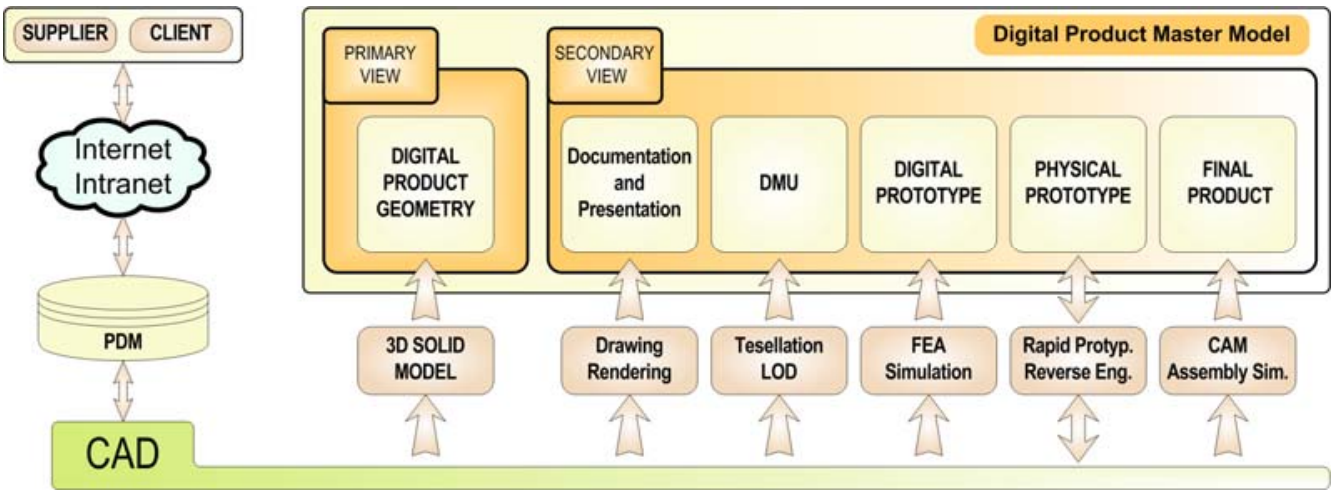

Figure 5. Digital Product Model.

tessellation and implement models with different levels of detail (LODs), specially for visualizing complex structures.

- Digital prototypes: the simulation and finite element applications use simplified representations of the primary view for making their calculations. FEA applications need geometry to be free of small details to proceed with mesh generation. This can be easily accomplished by an appropriate modeling methodology, where unwanted features can be suppressed.

- Physical prototypes: Hand-made prototypes are being replaced with Rapid Prototyping tools. RP machines make use of a derived model extracted by tessellation from the 3D solid. The STL format is the industry standard for this purpose. The other application for Physical Prototypes is CAD data input in styling applications, where 3D laser scanning devices provide clouds of points that later must be transformed to surfaces and imported into the CAD application.

- Final products: CAM and assembly simulation make an intensive use of the Primary View. Besides, sometimes it is necessary to make modifications in the original geometry. For example, in mold making, sometimes nominal part geometry must be modified to avoid warpage. This requirement introduces additional difficulties, because the reuse of the primary CAD model for this purpose depends on the modeling methodology previously used.

Finally the importance of the associativity concept must be highlighted, since allows changes made on the primary view to be automatically transferred to the secondary ones, avoiding many mistakes caused by the continuous variations suffered by the product model during the development process. The quest for associativity is also one of the reasons that justify the adoption of a unique integrated CAD system by big OEMs.

\section{Product Data Flow}

The diversity of partners and software tools, in the context of the Extended Enterprise, leads to a complex flow of product data. The hierarchical structure dominant in many industrial sectors, like the one shown in Figure 5, increases the complexity, because different data requirements, design responsibilities and CAX tools appear according to the considered tier. There are three alternatives for transferring product data in computer-readable form among the tiers [20]: use of a common system, direct translation or indirect translation by means of a neutral file. Current practices in industry for product data exchange among tiers will now be presented.

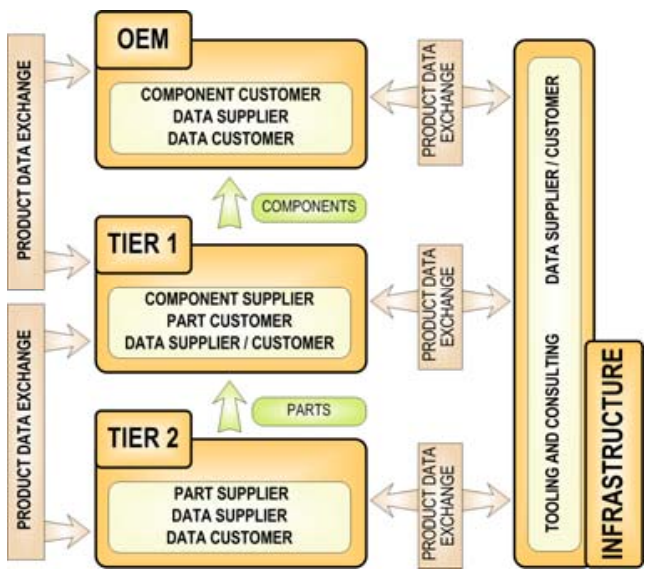

Figure 6. Tier structure of the automotive sector

\section{OEMs - Tier 1 Communication}

Original Equipment Manufacturers (OEM) in the automotive, aeronautical and aerospace industries oblige tiers 1 and 2 to achieve the technological level of "digital product" paradigm. To obtain the close integration of Tier 1 suppliers, OEMs force them to use the same CAD tools. A classical example is the alignment of each automotive maker with a specific CAD system. Thus, we have Ford and SDRC's Ideas, General Motors and UGS's Unigraphics and DaimlerChrysler and Dassault's CATIA. It is a conservative attitude, but is justified by the important role played by the main subcontractors, which are taking over the design of their 
components. Therefore, adopting the same CAD tools is recommended for a smooth integration with OEMs.

Some tasks in the upper tiers admit the use of neutral files. For example, current practice is to maintain a STEP version of files for storing purposes. In addition, some applications that do not need the feature representation, as Digital Mock-up, can support neutral files.

\section{Tier 1 - Tier 2 Communication}

A different situation appears at tier 2. In many cases, the same tier 2 subcontractor works for different tier 1 enterprises. Therefore, implementing the same CAX tools as all the upper tier partners is a very expensive approach.

These end-suppliers do usually not carry out design activity, because they only provide parts or simple assemblies. However, CAM applications are widely used. For this reason, traditional paper documentation, such as engineering drawings, has been replaced or complemented by 3D geometric models. In this situation, the data transfer alternatives are a direct translation strategy or use of neutral formats. The problems arise due to keeping translators updated when new software versions are released, and taking care of the loss of information contained in parametric and features definitions.

\section{The Infrastructure Tier}

In addition to providers previously considered in the vertical tier structure, another class of providers exists. They are called the infrastructure tier, and include tooling providers and engineering consulting firms. They can represent a challenging problem, because engineering and design consulting firms can help perform studies, like kinematical simulations or FEM analysis, where intensive and complex product data exchange and manipulation are normal issues. In other words, this tier has a high technological level, and provides specialized services to the other tiers. For example, the mold makers do both design and manufacturing activities, and use 3D modeling tools. Hence, being furnished with a geometric model is of great value to them, because they avoid the boring and error-prone task of modeling parts from drawings.

Most members of the infrastructure tier need to implement neutral file exchange. Economic reasons justify this solution, since these firms work for many different customers and the exchange of data has an important impact on cost. For example, a FEM consulting firm usually needs to build a simplified 3D model to perform its analysis. Its workload is different if they are furnished with a digital drawing, an IGES file with a surface representation, or a 3D model with feature information. In the latter case, for instance, sometimes an easy feature suppression operation provides the simplified model. This is an example showing that the pre-processing part of FEM analysis can be less time consuming if the proper representation is used in the input of design information. Therefore, it is very important to have a rich neutral format supporting features, constraints and parametric geometry.
As seen in the previous analysis, nowadays CAX tools require a continuous flow of data in the Product Development Process, where data exchange and its related standards play an important role.

\section{Standards Status}

The consolidation of ISO 10303 (STEP) as the main neutral format in industry has relegated IGES and other popular formats to a secondary role. The initial release of STEP published in 1994, provides a successful way to transfer both drawings and solid models. Nevertheless, current CAD systems provide modeling tools like parametric features, constraints and history-based modeling not supported by the current release of STEP. Consequently, the current edition of STEP can be said to provide a way of exchanging "static" information about the product. The information transmitted is simply a "snapshot" of the model, because when making the translation all the parameterization, constraints and feature information is omitted. This is a serious handicap for true collaborative engineering, because engineers encode their "design intents" in the selection of features, constraints and parameters they make. The proper nature of ISO standards development, based on a succession of stages (see Table 1), leads to a technological gap between current CAD systems and STEP capabilities.

\begin{tabular}{|l|l|l|}
\hline No. & Stage & Deliverable \\
\hline 0 & Preliminary & PWI. Preliminary Work Item \\
\hline 1 & Proposal & NWI. New Work Item \\
\hline 2 & Preparatory & WD. Working Draft \\
\hline 3 & Committee & CD. Committee Draft \\
\hline 4 & Enquiry & DIS. Draft International Standard \\
\hline 5 & Approval & FDIS. Final Draft International Std. \\
\hline 6 & Publication & IS. International Standard \\
\hline
\end{tabular}

\section{Table 1. Stages in ISO standards development}

However, there are several initiatives directed to shorten this gap. In 2001 two Application Protocols (AP) supporting features representation have reached IS status: AP214 and AP224. From a design point of view, AP 214 provides two Conformance Classes (CC14 and CC15) supporting feature based design. Besides, in the short term [18] STEP is expected to implement 2D parametric sections (explicit geometry supporting different types of geometric, numeric and algebraic constraints) and 3D parametric assemblies (connecting associations among the components constituting an assembled product and their relations). This is possible because they are relatively easy to implement with the current structure of STEP. For that, new Integrated Generic Resources (part 42, 50 and 51) and Integrated Application Resources (part 108 and 109) are being implemented.

The support for history-based modeling is a more complex task. These kinds of models store the sequence of modeling operations used to build them. Therefore, it is necessary to define operators to create, query and modify geometric entities. To implement these operators it is necessary to define a generic application programming interface (API) that can be supported by commercial CAD applications, and a 
representation of the operators in STEP. The definition of this generic API can take previous projects as reference, such as CAM I, standards as ISO 13584 (Parts Library) and new initiatives such as the Request for Proposal "CAD Services" [19] submitted by the Object Management Group (OMG).

\section{Product Data Quality}

The growing importance of product data exchange for the product development process in the context of the extended enterprise has been analyzed in previous sections. The lack of standards supporting the capabilities of current commercial $\mathrm{CAD}$ applications, the variety of downstream applications making use of product data and the heterogeneous nature of work teams leads to an increasing interoperability problem. This supposes repeatedly wasting time and money, every time fixing or rebuilding poor data quality models is needed.

A study done by ERIM [24] concluded that poor data quality adds $10 \%$ to the cost and up to $25 \%$ to delivery time in the U.S. tooling industry. A survey performed by the NIST Strategic Planning and Economic Assessment Office in 1999 [21], estimates the economic cost of bad interoperability in the U.S. automotive industry at one billion dollar per year. A similar study in the German automotive industry [22] calculates approximately in half billion dollar per year the economic impact of the data exchange problem.

To provide answers to this problem, we must distinguish between intrinsic and extrinsic problems related to the data exchange process. Intrinsic problems are those related to the structure of the $\mathrm{CAD}$ model before any translation process begins, while extrinsic problems are related to those issues appearing during translation. In this paper, we are going to focus on the intrinsic aspect of the product data exchange problem. Here, is where the concept of product data quality is fundamental to understand the origin of many problems that suppose impediments to collaborative engineering. Extrinsic problems, which have been extensively studied in the literature (see, for example, Vergest and Horváth [25]), are not considered for the present study.

\section{Data Quality Definitions}

The Automotive Industry Action Group (AIAG) defines product data quality in the following way:

"Quality Product Model Data is constructed accurately, completely representing the geometric model (math data), and accurately and completely representing all additional information in a way that can be shared and used by multiple users and managed with a minimum effort".

However, Phelps [25] proposes a more simple definition: "Product data quality is a measure of the accuracy and appropriateness of product data combined with the timeliness with which those data are provided to all the people who need it". This definition is close to the concept of data quality coming from the Software Engineering domain, where a list of desirable quality dimensions is defined. For example, Ballou and Pazer [27] identify four dimensions of data quality: accuracy, completeness, consistency and timeliness.
Other approaches give a wider vision integrating contextual aspects of data quality. Thus, Shanks [28] proposes a semiotic data quality framework based on four levels: the syntactic (structure of data), the semantic (meaning of data), the pragmatic (usage of data) and the social level that concerns the shared understanding of the meaning of symbols.

Another important idea commented by Wand and Wang [29] is that the notion of data quality depends on the actual use of data. They agree with many other authors that define data quality as "fitness for use", showing that the concept of data quality is relative. Finally, they also note that as important as defining the concept of data quality is to know how it is to be measured.

\section{Product Data Quality Standards}

At present, the most extended product data quality standard is VDA 4955 [30] and its equivalent ODG11CQ9504 "ODETTE CAD/CAM Quality Assurance Method" ODETTE standard. Although it has its origins in the automotive sector, it has been adopted in many other industries. VDA 4955 provides quality criteria for both geometrical and organizational aspects of CAD/CAM data. These criteria can be implemented in software applications, known as quality checkers, to automate quality auditing.

The geometric criteria analyze polynomial degree of curves and surfaces to avoid undesired oscillating curves and rippling surfaces. There are criteria for checking the orientation and parametrization of curve elements and surfaces. The detection of surface and curve defects (overlaps, steps and gaps) and the analysis of their continuity is very important for downstream applications such as NC processing and coordinates measuring machines (CMM). The organizational criteria of VDA 4955 propose some recommendations related to model naming and structuring, drawing generation and modeling methodology.

Other organizations in the automotive industry have developed similar standards. Thus, the French association "Groupement pour l'Amélioration des Liaisons dans l'Industrie Automobile" (GALIA) has developed the standard CAO.3 y CAO.4 with similar content to VDA 4955. The Japan Automotive Manufacturers Association (JAMA) has recently developed a standard related to product data quality. In the U.S., the "Automotive Industry Action Group" (AIAG) has established its Vehicle Product Data Quality (VPDQ) work group after the organization identified product data quality as the highest priority issue affecting product development in supply chains.

With the objective of unifying the emergent national recommendations related to product data quality, the "Strategic Automotive product data Standards Industry Group" (SASIG), established in 1995, is working on an international recommendation (SASIG-PDQ) for Product Data Quality in the automotive industry. This group comprises AIAG, VDA, GALIA, JAMA, ODETTE Sweden, Australia's Federal Chamber of Automotive Industries (FCAI) and the Japan Automobile Parts Industry (JAPI). The goal is to release an initial version of this recommendation in 2002. 
Other interesting references to PDQ standards can be found in OEMs web sites dedicated to suppliers, for example:

- BMW: http://www.zulieferer.bmw.de

- General Motors: http://www.gmsupplypower.com

- Volkswagen: http://www.vw-zulieferer.de

- DaimlerChrysler: http://supplier.daimlerchrysler.com

- Ford: https://fsn.ford.com

\section{A Linguistic Model of Product Data Quality}

After a detailed analysis of precedent standards on product data quality, various "levels of quality" have been found in a clear parallelism with the different levels and approaches that natural language analysis use: phonology (phonetics and sounds), morphology (forming words from more basic meaning units), syntax (forming sentences out of words), semantics (sentence meanings obtained from words) and pragmatic (understanding how sentences are used).

Therefore, three levels of quality can be distinguished:

1. Morphological quality.

2. Syntactic quality.

3. Semantic/pragmatic quality.

The morphological quality is related to the geometrical and topological correctness of the CAD model. The syntactic quality evaluates the use of the proper modeling conventions. The semantic/pragmatic level takes into account the CAD model capability for reusing and modification.

These levels of quality are closely related to the proposed product data model, where we have defined both primary and secondary views of product model. A primary view of high quality avoids many of the problems that appear during the product data exchange process. It is evident that in this context the "fitness for use" concept is clearly applicable, because the secondary views of the model need data of different quality according to the data's application. Thus, some secondary views only require a primary view of good morphological quality. They are simplified geometric representations of the primary view, where the usability depends on the geometric and topological correctness. This is the case of DMU and presentation views.

In other situations, where sharing product data is important, "something more" than correct geometry is needed. Additional organizational information must be understood, for instance, naming conventions, layer structure, parameters and more attributes related to the syntactical quality of the model. These situations cover the need of accessing the model without proceeding for modifications. This is the case of the documentation view where it is important to follow some modeling conventions.

Finally, when accessing a model for modification is required, additional quality is needed. Many CAD users have suffered from a regeneration error, due to a modification of a simple dimension in a complex solid model with hundreds of features. This is the level associated to semantic/pragmatic quality, where the modeling methodology is the key element to success in reusing models.

The recommendations presented in previous sections, as in VDA 4955, provide criteria for the morphological and syntactic levels. These two levels have been the object of detailed study in recent years. Nowadays commercial applications dedicated to automating the verification of the quality criteria in CAD models do exist, although the offer is still reduced. The syntactic level depends heavily on in-house modeling conventions. In industrial sectors where few OEMs control the market, such as the automotive and aeronautical sectors, the syntactic quality criteria plays an important role in the smooth communication within the work team inside the extended enterprise framework. Information related to the semantic quality level is hard to find, because the modeling methodologies that provide the criteria for semantic/pragmatic quality belong to the enterprise's know-how. It is related to the domain of Knowledge Management, so it has a strategic value, and remains hidden to public diffusion. In the following sections, a detailed vision of each quality level will be given.

\section{Morphological Level}

VDA 4955 provides a good reference for assessing the morphological quality of a CAD model. In Table 2 , a compact relation of the geometric criteria provided by this recommendation is presented. The geometry checks provide information on how and with what exactness geometry elements are generated, to ensure the subsequent usability of these elements within the process chain. These checks are coded according to their type: wire geometry (M \& $\mathrm{C}$ ); surface (SU); faces in bounded surfaces (F); surface topology (T); B-rep solids (SO), and drawing elements (D). Geometric defects are usually due to bad modeling practice or incorrect CAD application algorithms. Round-off errors are responsible for many problems. The choice of the accuracy parameter is very important too. Most of CAD systems in the market provide some of the following accuracy types:

- Relative accuracy: the smallest element or the largest gap is in proportion to the model-bounding box.

- Adjustable absolute accuracy: the user fixes the size of the smallest element or largest gap.

- Fixed absolute accuracy: the user cannot modify the accuracy value.

Industry best practices recommend defining a common absolute accuracy to avoid data exchange problems.

\begin{tabular}{|l|l|l|}
\hline Code & Description & Criteria \\
\hline M2 & Identical elements & $>0.02 \mathrm{~mm}$ \\
\hline SU9 & Min. curvature radius & $>0.5 \mathrm{~mm}$ \\
\hline F15 & Distance to its surface & $<0.02 \mathrm{~mm}$ \\
\hline T18 & No. of faces per edge & $<3$ \\
\hline SO26 & Multi-body solids & No \\
\hline D28 & IGES conform texts & Yes \\
\hline C7 & Distance to itself & $>0.02 \mathrm{~mm}$ \\
\hline
\end{tabular}

Table 2. Some geometry checks in VDA 4955/2 


\section{Syntactical Level}

Modeling conventions are a basic issue to avoid data sharing problems and provide an easier understanding of CAD models. Modeling conventions are usually implemented through a series of configuration files and start parts, assemblies and drawings. In most cases, OEMs provide their suppliers with some documentation about preferred modeling conventions. For an effective CAD implementation the first step is to define a convention guide and their support files and then to enforce its use thoroughly. Many modeling conventions can be automated by means of configuration files and the use of macros, and some quality checkers support this kind of "syntactical analysis". The analysis of the modeling conventions issued by some of the main automotive OEMs indicates that those included in Table 3 are the most widely employed.

\begin{tabular}{|l|}
\hline Modeling conventions \\
\hline Units. \\
\hline Coordinate systems. \\
\hline Naming conventions. \\
\hline Layer structure and function. \\
\hline Part/assembly parameters and attributes. \\
\hline Engineering change representation. \\
\hline Content of simplified representations. \\
\hline Drawing characteristics. \\
\hline Tolerance settings. \\
\hline
\end{tabular}

\section{Table 3. Frequent modeling conventions}

The successful management of complex engineering projects, where many different people share product data, requires the application of these modeling conventions to be enforced. As the major part of these conventions are enterprise-dependent, syntactical quality checkers provide a basic framework that must be customized to serve the directives of each enterprise. This quality level has an important relation with PDM systems, because many of the conventions are used to find parts and assemblies in the database. In this case, naming conventions and part/assembly parameters and attributes achieve special importance.

\section{Semantic/pragmatic Level}

The semantic/pragmatic level takes into account the CAD model capability for reusing and modification. CAD users have a great variety of modeling tools for shaping their designs. However, experience shows that certain procedures provide better solutions than others. This knowledge is very important, and must be documented for it to be easily accessible. Many enterprises have developed their own internal "modeling guidelines" where the "best practices" for improving CAD model quality are recorded.

In the context of the Extended Enterprise, the modeling methodology is more important than in the traditional product development process. Our experience in previous projects [31] confirms that the integration of first tier suppliers in the definition of the modeling guidelines is very interesting. A clear example is molding makers that as final suppliers have both design and manufacturing tasks. Sometimes, nominal part geometry must be deformed to allow injected parts to get the right geometry and tolerances. The needed deformation must be generated in the 3D model before NC programming. There are always several modeling alternatives to get the desired part geometry, and only some of them allow later modifications required for the mold design to be made. The mold supplier has his own point of view on the problem, which forces him to modify the 3D model in a very specific way. For this reason, in this example, it is very important to provide a communication channel between OEM and mold maker, because if both partners share the right modeling technique, time and cost to develop molds can be reduced in a dramatic way.

The previous analysis serves to introduce a new concept that we named extended modeling [31]. We define "extended modeling" as a modeling methodology that integrates different perspectives from the product development process in the frame of the Extended Enterprise Collaboration. The use of a common modeling strategy between partners will permit the reuse of the generated model and the improvement of the effectiveness of downstream applications within third tier suppliers. Including suppliers in the development of the modeling guidelines allows them to concentrate on added value tasks, instead of wasting time reintroducing or adapting the geometric information they receive. In this way, an analogy can be established with the Design for $\mathrm{X}$ methodologies (DFx), with a Modeling for X, where X means molding, manufacturing, analysis, and so on.

In the previous section, the choice of those features appropriate to facilitate downstream operations has been a key element. However, another important aspect of semantic quality is the structure of the CAD model. Complex parts with more than one hundred features become difficult to modify because of the multiple interrelations among features. Without a careful working procedure, undesired dependencies can appear. Anderl and Mendgen [32] provide interesting considerations on this matter. They represent parent/child feature relations in a matrix form, and try to reorganize the model to approximate this matrix to its diagonal form. Thus, related features are grouped and many unwanted dependencies are avoided. Current CAD systems provide limited support for analyzing features dependencies. Usually a tree representation is used to display the modeling history, but it is difficult to achieve a whole vision of the dependencies in the model.

\section{Implementation}

To implement a strategy on product data quality (PDQ), it is important to adhere to some PQD standard, such as VDA 4955 , that provides a good reference for analyzing morphological quality and develop modeling conventions and modeling guidelines adapted to the product development process.

Commercial quality checkers provide a valuable help for enforcing morphological and syntactical checks. Usually, many modeling conventions are implemented by means of a 
set of configuration files and start parts, assemblies and drawings. These elements combined with a quality checker ensure that the desired syntactical quality level is obtained.

Most of the quality checker applications are based on Web browser technology, where recommendations are presented to the user in the form of an HTML, XML and Java based report, which appears in the user's Web browser.

The quality evaluation process must be done before exchanging models with other CAD system or submitting them to the PDM server. Commercial tools support both interactive and batch process to automate the checking process. The growing number of applications dedicated to this task is a clear indicator showing an industry need for improving the quality of models. The most widely used checkers are:

- Parametric Technology: ModelCHECK.

- International TechneGroup Incorporated: CAD/IQ.

- Prescient Technologies: DesignQA.

- Software Factory: PE Check.

- RAND TransCAT: Q-check.

Web technology is also a good communication channel to circulate modeling conventions and guidelines. In this way, they can be made available through the enterprise web site, where selected suppliers can have authorization to enter the restricted access areas where these documents are stored.

\section{Conclusion}

The expansion of concepts like extended enterprising and collaborative engineering is forcing an exponential growth of data flow inside the product development team. Product Data Quality is a key issue to avoid data exchange problems and simplify the integration of downstream applications in the design chain.

The proposed linguistic approach to PDQ tries to clarify concepts present in the current Product Data Quality literature and emphasizes the role of modeling methodology to achieve high quality models. Even though the morphological and syntactical levels are quite developed, the evaluation of semantic quality remains an open issue in quality checker applications. It is a complex task, where new research activity must provide tools to evaluate objectively the semantic quality of models.

Modeling methodology is an important but poorly documented topic. Best practices documents are difficult to find because much of enterprise expertise is embedded in the modeling strategy. Therefore, semantic/pragmatic quality procedures become a strategic know-how that industry preserves and does not publicize. The "extended modeling approach" transfers this knowledge inside the extended and/or virtual enterprise, providing overall lower cost and shortening the development time.

Finally, the importance of training to reach a high quality level in PDQ must be noted. The best CAD system in the world used by a badly trained operator without a good modeling methodology produces bad CAD models that impede the effectiveness of downstream applications. Therefore, it is as important to invest in training as to have good data exchange standards and detailed modeling methodologies and conventions. Training has a direct impact in rising overall productivity and shortening development time. Our experience shows that the development of "modeling guidelines" has also proved itself to be an important aid for training purposes. New users have developed good modeling skills and become productive in less time than by previous training methods.

\section{Acknowledgements}

This work has been partially sponsored by the Spanish Government national R\&D FEDER program. Project No. 1FD97 0784 "Implementing Design and Manufacturing Advanced Technologies in a Concurrent Engineering Environment. Application to an Automotive Components Manufacturing Company". Thanks are also due to Radiadores Ordoñez S.A., whose collaboration has been very important to check the approach effectiveness.

\section{References}

1. R.J. Winner, J.P. Pennell, H.E Bertrand and M.M. Slusarczuk, The role of concurrent engineering in weapons system acquisition, IDA R-338, Institute for Defense Analyses, 1988

2. K.J. Cleetus, "Definition of Concurrent Engineering" CERC Technical Report Series, CERC-TR-RN-92-003, Concurrent Engineering Research Center, West Virginia University, Morgantown, 1992.

3. Bates, H. and D. Twigg, "Outsourcing of Automotive Component Design", 30 ${ }^{\text {th }}$ Int. Symposium for Automotive Technology and Automation, Florence, June 1997

4. M. Hardwick, R. Bolton, "The industrial virtual enterprise", Communications of the ACM, Vol. 40, No. 9 1997, pp. 59-60.

5. Glossary of the European Society of Concurrent Engineering, available online at $<\mathrm{http}: / / \mathrm{www}$.esoce.net/Glossary.htm>, 2001.

6. K.D. Thoben, F. Weber, "Information and Communication Structures for Product Development in the Concurrent Enterprise: Requirements and Concepts", ASI'97, The Annual Conference of ICIMS-NOE, Budapest, July $14^{\text {th }}-18^{\text {th }}, 1997$, pp. 460-467.

7. R. de Graaf, Assessing Product Development, Visualizing Process and Technology Performance with RACE 2. Ph.D. Thesis, Eindhoven University of Technology, The Netherlands, available online at $<\mathrm{http}: / /$ www.tm.tue.nl/race/race2>, 1996

8. S. Pugh, Total design: integrated methods for successful product engineering, Add.-Wesley, Amsterdam, 1991.

9. G. Boothroyd, P. Dewhurst and W. Knight, Product design for manufacture and assembly, Marcel Dekker, New York, 1994.

10. P. Klingstam , P. Gullander, "Overview of Simulation Tools for Computer-Aided Production Engineering", Advanced Summer Institute - ASI'97, Budapest, Hungary.

11. T. Bilgiç, D. Rock, "Product Data Management Systems: Stateof-the-art and the Future", Proceedings of DETC'97 ASME Design Engineering Technical Conference, September 14-17, 1997, Sacramento CA. 
12. E. Miller, "Managing Product Data throughout the Life Cycle" Computer-Aided Engineering, march 2000, available online at $<$ http://www.caenet.com/res/archives/200003cimsup.html $>$.

13. Z. Deng, J. Pettersen, E. N. Jensen, B. Bang, and R. Davidrajuh: "Realizing Inter-enterprise Remotely Synchronous Collaborative Design and Planning within Multi-platform Environment". Proceedings of the $7^{\text {th }}$ ISPE International Conference on Concurrent Engineering (CE2000), Lyon France, July 2000

14. B.R. Höhn, K. Steingröver and A. Dyla, "Computer Aided Product Development". Proceedings of the International Design Conference - DESIGN 2000, Dubrovnik, May 23-26, 2000, pp. 307-312

15. VDA Recommendation 4953, part 1: Simplified CAD Drawing. VDA e.V., Frankfurt, Germany, 1999.

16. C.M. Hoffmann, R.J. Arinyo, "Distributed maintenance of multiple products views". Computer-Aided Design Vol. 32 (2000) pp. 421-431.

17. CAD/CAM Data Exchange with Volkswagen, version 2.3, available online at <http://www.vw-zulieferer.de/cad_komp/ $\mathrm{acad} /$ English/cad cam data eng.pdf $>, 2001$

18. A. Ohtaka, "Parametrics: Present and future" Proceedings of the $5^{\text {th }}$ International Conference on Information Systems Analysis and Synthesis ISAS'99, July 31- August 04, 1999, Orlando, Florida.

19. CAD Services V1.0, (OMG document mfg/2001-06-03) available online at <http://cgi.omg.org/cgi-bin/doc?mfg /00-06-07>, 2001.

20. M.S. Bloor, J. Owen, Product Data Exchange, UCL Press, London, UK, 1995.

21. NIST. Interoperatibility cost analysis of the US automotive supply chain, NIST Strategic Planning and Economic Assessment Office, Planning Report \#99-1, available online at $<$ http://www.nist.gov/director/prog-ofc//report99-1.pdf>, 1999

22. D. Trippner, M. Endres, STEP - The Significance for the Designer, Product Data Journal, No. 2, 1998, pp. 13-15

23. S. Szykman, S.J. Fenves, W. Keirouz, S.B. Shooter, "A foundation for interoperatibility in next-generation product development systems", Computer-Aided Design Vol. 33, pp. 545-559, 2001

24. ERIM, Center for Electronic Commerce, CAD/CAM Data Problems and Costs in the Tool and Die Industry, available at $<$ http://www.erim.org/cec/paperscadcam_exec.htm>, 1991

25. T. Phelps, "Extending Quality Concepts to Product Data", AIAG Actionline, Vol. 19 No. 7, August 1999, pp. 38-42,

26. J.S.M. Vergeest, I, Horváth, "Where interoperatibility ends", Proc. of the 2001 Computers and Information in Engineering Conference, DETC'01, ASME, New York, 2001, available online at $<$ http://dutoa36.io.tudelft.nl/docs/cie21233.pdf>, 2001

27. D.P. Ballou, H.L. Pazer, "Modeling data and process quality in multi-input, multi-output information systems", Management Science Vol 31, No. 2 (1985), pp. 150-162

28. G. Shanks, B. Corbitt, "Understanding data quality: social and cultural aspects". Proc. $10^{\text {th }}$ Australasian Conference on Information Systems, 1999, pp. 785-797, available online at $<$ http://www.vuw.ac.nz/acis99/Papers/PaperShanks-095.pdf>, 1999.

29. Y. Wand and R.Y. Wang, "Anchoring data quality dimensions in ontological foundations", Communications of the ACM, Vol. 39, No. 11 (Nov. 1996), pp. 86-95.

30. VDA Recommendation 4955/2: Scope and Quality of CAD/CAM Data. VDA e.V., Frankfurt, Germany, 1999

31. C. Vila, M. Contero and P. Company, "Extended modelling, a tool for cooperative design". Proceedings of the $6^{\text {th }}$
International Conference on Concurrent Enterprising, ICE 2000, june 28-30, 2000, Toulouse France, pp. 171-179, 2000

32. R. Anderl, R. Mendgen, R.: "Analyzing and Optimizing Constraint-Structures in Complex Parametric CAD Models",. Geometric Constraint Solving and Applications, SpringerVerlag, Germany, 1998. 